\title{
Susceptibility to Peer Influence in Adolescents with Mild-to-Borderline Intellectual Disability: Investigating Links with Inhibition, Theory of Mind and Negative Interpretation Bias
}

Eline Wagemaker ${ }^{1,2}$, Tycho J. Dekkers ${ }^{1,3,4,5}$, Anika Bexkens ${ }^{6,7}$, Elske Salemink ${ }^{8}$, Jacqueline N. Zadelaar $^{9}$, Hilde M. Huizenga ${ }^{1,2,10}$

${ }^{1}$ University of Amsterdam, Department of Psychology, Amsterdam, The Netherlands

${ }^{2}$ Research priority area Yield, University of Amsterdam, Amsterdam, The Netherlands

${ }^{3}$ Levvel, Academic Center of Child- and Adolescent Psychiatry and Specialized Youthcare, Amsterdam, The Netherlands

${ }^{4}$ University Medical Center Groningen, Department of Child and Adolescent Psychiatry, Groningen, The Netherlands

${ }^{5}$ Amsterdam University Medical Center, Department of Child and Adolescent Psychiatry, Amsterdam, the Netherlands

${ }^{6}$ Leiden University, Department of Psychology, Developmental and Educational Psychology, Leiden, The Netherlands

${ }^{7}$ GGZ Delfland, Delft, The Netherlands

${ }^{8}$ Utrecht University, Department of Clinical Psychology, Utrecht, The Netherlands

${ }^{9}$ Vrije Universiteit, Department of Educational and Family Studies, Amsterdam, The Netherlands

${ }^{10}$ Amsterdam Brain and Cognition, University of Amsterdam, Amsterdam, The Netherlands

Corresponding author: Eline Wagemaker, University of Amsterdam, Department of Psychology, Nieuwe Achtergracht 129b, 1018 WS Amsterdam, The Netherlands. Telephone: 


\begin{abstract}
Background: Adolescents with mild-to-borderline intellectual disability (MBID) may be more susceptible to peer influence than typically developing (TD) adolescents.
\end{abstract}

Aims: This preregistered study compares adolescents with MBID and TD adolescents on their susceptibility to peer influence and investigates links with three potential underlying mechanisms: inhibition, Theory of Mind (ToM) and negative interpretation bias.

Methods and Procedures: We assessed 163 adolescents (111 MBID, 52 TD; 14-19 years; $63 \%$ boys) using experimental tasks and self- and/or teacher-reports.

Outcomes and Results: Adolescents with MBID and TD adolescents did not differ in their susceptibility to peer influence, inhibition and negative interpretations. On two of the four ToM instruments, adolescents with MBID showed weaker ToM than TD adolescents. In a structural equation model, tested in the MBID group, the latent factors inhibition, ToM and negative interpretation bias were not related to susceptibility to peer influence.

Conclusions and Implications: Using a multimethod approach, a structural equation model, and a large sample, this study revealed new insights in adolescents with MBID: they did not differ from TD adolescents in many domains and their inhibition, ToM and negative interpretation bias were not linked to their susceptibility to peer influence.

\title{
What this paper adds?
}

The current study offers three novel findings in understanding susceptibility to peer influence in adolescents with mild-to-borderline intellectual disability (MBID). First, we demonstrated that adolescents with MBID did not differ from typically developing (TD) adolescents 
regarding their susceptibility to peer influence. As earlier studies did find heightened susceptibility to peer influence in adolescents with MBID, we argue that this susceptibility may depend on specific conditions in which it is studied. Second, adolescents with MBID had to some extent weaker Theory of Mind (ToM) than TD adolescents, whereas their inhibition and negative interpretation bias were not different. Third, we showed that the susceptibility to peer influence of adolescents with MBID is not linked to their inhibition, ToM or negative interpretation bias. Next to these findings, this paper also adds to the literature in a methodological way, as it is a preregistered study using a multimethod approach to measure all constructs, implements instrument selection/adaptation based on explicit criteria, and uses a structural equation model with latent factors to test the full theoretical model.

Keywords: Adolescence; Peer Influence; Intellectual Disability; Inhibition; Theory of Mind; Negative Interpretation Bias

Word count: 8000 


\section{Introduction}

Adolescence has generally been characterized by higher susceptibility to peer influence (see Do et al., 2020 for a review). Adolescents with mild-to-borderline intellectual disability (MBID) may be even more susceptible to the influences of their peers. MBID is highly prevalent: among children and adolescents around ten percent meet the criteria of mild problems in conceptual, social and adaptive skills of functioning and an IQ between 50 and 85 (American Psychiatric Association, 2013; Simonoff et al., 2006). Increasing scientific evidence shows that adolescents with MBID are more susceptible to peer influence than typically developing (TD) adolescents (Bexkens et al., 2019; Dekkers et al., 2017; Egger et al., 2021; Wagemaker et al., 2020). However, it is still unknown why this may be the case. Therefore, we aim to gain a deeper understanding of susceptibility to peer influence in adolescents with MBID. Here, we compare adolescents with MBID and TD adolescents on their susceptibility to peer influence and link their susceptibility to peer influence to potential underlying factors.

\subsection{Susceptibility to peer influence in adolescents with MBID}

Heightened susceptibility to peer influence in adolescents with MBID has been shown in multiple studies using different measurement methods. Two experimental studies demonstrated that adolescents with MBID took more risks than TD adolescents in a computer task with virtual peers encouraging risk taking (Bexkens et al., 2019; Wagemaker et al., 2020). Another study found that adolescents with MBID more often changed their judgement about popularity of a person based on judgements of their virtual peers than TD adolescents (Egger et al., 2021). Moreover, adolescents with MBID reported less resistance to peer influence than TD adolescents (Dekkers et al., 2017). In the current study we add to these 
previous findings by administering an experimental task and self-report of susceptibility to peer influence.

\subsection{Factors linked to susceptibility to peer influence in adolescents with MBID}

To understand why adolescents with MBID may be susceptible to peer influence, we investigate several potential factors. A recent review on neural biomarkers of susceptibility to peer influence in adolescence indicates that psychological processes that encode regulatory, social-cognitive, and affective cues from the environment contribute to peer influence susceptibility (Do et al., 2020). We operationalize these three processes into three factors: inhibition, Theory of Mind (ToM) and negative interpretation bias.

1.2.1 Inhibition. Inhibition refers to all processes that enable deliberate suppression of dominant responses to serve higher-order or longer-term goals (Nigg, 2000). Stronger inhibition at age 12 in TD adolescents predicts lower susceptibility to peer influence three years later (Meldrum et al., 2013), and a meta-analysis demonstrated that individuals with MBID have a medium to large inhibition deficit compared to TD adolescents (Bexkens, Ruzzano, et al., 2014). Therefore, we hypothesize that inhibition problems in adolescents with MBID may lead them to impulsively going along with peers.

1.2.2 ToM. ToM refers to the ability to attribute mental states such as beliefs, desires, emotions, and intentions to oneself and others (Humphrey \& Dumontheil, 2016). Better ToM protects against binge drinking under peer influence (Laghi et al., 2019). Adolescents with MBID perform worse on ToM tasks relative to TD adolescents (Baglio et al., 2016). Therefore, we hypothesize that problems with ToM in adolescents with MBID may lead to unawareness or misunderstanding of peers' intentions which can result in following advice from peers. 
1.2.3 Negative interpretation bias. A negative interpretation bias is the tendency to interpret ambiguous social cues as signs of rejection (Stuijfzand et al., 2018). This bias is often present in TD adolescents with high social anxiety and increases fear of social exclusion (Miers et al., 2008). This fear of social exclusion may increase susceptibility to peer influence by (a) reputation management: avoid rejection by peers (Brechwald \& Prinstein, 2011) or by (b) increased stress in peer situations (Gunther Moor et al., 2014). Although never studied before, adolescents with MBID may have a stronger bias than TD adolescents because social anxiety disorder is more common in adolescents with MBID than in TD adolescents (Dekker \& Koot, 2003), and higher levels of social anxiety have been linked to higher negative interpretation bias in adolescents with MBID (Klein et al., 2018). Therefore, we hypothesize that a strong negative interpretation bias in adolescents with MBID may increase their susceptibility to peer influence.

\subsection{The current study}

This preregistered study adopted a multimethod approach with experimental tasks, self-reports and/or teacher-reports for all constructs. A multimethod approach enhances reliability and validity (Brewer \& Hunter, 2006), which is especially important in MBID populations, as correlations between different instruments have been reported to be low for this group (e.g., Bramston \& Fogarty, 2000). Our first aim was to investigate group differences between adolescents with and without MBID in their susceptibility to peer influence, inhibition, ToM, and negative interpretation bias on all instruments. Our second aim was to investigate how inhibition, ToM, and a negative interpretation bias are linked to susceptibility to peer influence in adolescents with MBID using structural equation modelling (SEM). In this analysis, we estimate latent factors underlying multiple instruments (e.g., experimental task and self-report), which is beneficial as it corrects for noise and thus has 
higher reliability compared to the instruments themselves (Schreiber et al., 2006). We also estimate the relationships between these latent factors (i.e., susceptibility to peer influence on the one hand and inhibition, ToM and negative interpretation bias on the other). As opposed to multiple analyses, testing this in one analysis is beneficial because it considers the influence of all factors. 


\section{Method}

The current study was preregistered in two parts (see https://osf.io/2mu6v and https://osf.io/vjb92). We followed the preregistrations, yet we did not entirely reach planned sample size due to COVID-19 related necessity of ending data collection. The anonymized data and syntax can be found on: https://osf.io/qey3a/.

\subsection{Participants}

Participants were 163 adolescents, ages $14-19$, with $(N=111)$ and without $(N=52)$ $\mathrm{MBID}^{1}$ (see Table 1 for group characteristics). The MBID group was sampled from practical vocational track schools. In the Netherlands, these schools have the following admittance criteria: an IQ between 55 and 80 on a standardized IQ test measured no more than two years prior to admittance and learning delays of $50 \%$ or more in at least two educational areas (i.e., mathematics, reading accuracy and fluency, reading comprehension, and spelling). The TD group was recruited at regular schools from the lower and higher general secondary education level and from the intermediate vocational education level.

To ensure suited IQ, we only included adolescents with an IQ between 50 and 85 in the MBID group and adolescents with an IQ higher than 85 in the TD group. Because of time constraints, we did not use the level of adaptive functioning as an inclusion criterion. Our sample was representative as we did not exclude any forms of psychopathology, which are highly prevalent (around 40\%) in MBID (Dekker \& Koot, 2003). Nevertheless, we administered short screeners for adaptive functioning and psychopathology to compare the groups. Parents provided active informed consent and adolescents provided assent. This study was approved by the local ethical review board.

- Insert Table 1 here -

\footnotetext{
${ }^{1}$ The MBID group was larger than the TD group, to allow for SEM within the MBID group.
} 


\subsection{Materials}

2.2.1 Descriptive measures. To characterize the groups, we assessed intelligence using the shortened Wechsler Intelligence Scale for Children (WISC-V; Na \& Burns, 2016) or Wechsler Adult Intelligence Scale (WAIS-IV; Wechsler, 2008), socio-economic status (SES; UNESCO, 1999), adaptive functioning using the Assessment of Social Adaptability - Youth teacher report (BSA-J; Lekkerkerker \& Konijn, 2011), and psychopathology using the Strengths and Difficulties Questionnaire self-, parent- and teacher reports (SDQ; Goodman, Ford, Simmons, Gatward, \& Meltzer, 2000). See Supplement A part I for details.

- Insert Table 2 here -

2.2.2 Multimethod approach. Susceptibility to peer influence, inhibition, ToM, and negative interpretation bias were assessed with an experimental task, self-report and/or teacher report (see Table 2). We selected experimental tasks based on the following criteria: minimal verbal requirements, minimal working memory requirements, minimal ceiling effect in the TD group, highly motivating, norms available, and validated in more than one study. As self-report can be difficult for adolescents with MBID (Emerson et al., 2013), we added comprehension checks to the online questionnaires (see Supplement A part II). We did not include teacher reports on susceptibility to peer influence and negative interpretation bias, as no instruments were available. More detailed information on the instruments, the psychometric qualities, and earlier use in the MBID population can be found in Supplement A parts III to VIII.

\subsubsection{Susceptibility to peer influence.}


2.2.3.1 Experimental task. In the Balloon Analogue Risk Task (BART; Lejuez et al., 2002 adapted from Dekkers et al., 2020b) adolescents were instructed to earn money by pumping a virtual balloon. Each pump was worth $€ 0,01$; but the balloon could explode at each next pump which meant that the money for that balloon would be lost. Adolescents could 'cash' the balloon at any point. This money remained in possession of the adolescent. The BART consisted of 30 test trials divided over two blocks with a break in between. The explosion points were determined randomly ranging between 5 and $128(M=64$ for both blocks), the same array of explosion points was used for every adolescent. We included two practice blocks with three balloons each to practice pumping and cashing. The practice blocks had identical mean explosion points as the experimental blocks.

Adolescents performed the BART twice: in a solo condition as described above and in a peer condition where they received advice from a peer. In the peer condition, before the task started, adolescents were told that a sex- and age-matched peer from another school would try to predict their performance. This peer was watching the adolescent via a camera positioned behind. In fact, the peer was a confederate of the study. To increase credibility of the peer, there was a short, standardized introduction to the peer via WhatsApp on a smartphone that was provided to the adolescent. At the beginning and halfway the task, during a break, adolescents received negative risk encouraging messages from the peer (i.e., "you pump very little")2. The difference in the number of pumps between the BART peer and solo was used as an indication of susceptibility to peer influence.

2.2.3.2 Self-report. The Resistance to Peer Influence Scale (RPI; Steinberg \& Monahan, 2007) consisted of ten questions using a tree-based structure. The outcome was the total score, higher scores indicated more resistance to peer influence. To obtain an index of susceptibility to peer influence, RPI total scores were multiplied by -1 .

\footnotetext{
${ }^{2}$ In the BART solo, adolescents watched two short neutral aquarium movies on the research phone at these moments to control for any distractions by the research phone.
} 


\subsubsection{Inhibition.}

2.2.4.1 Experimental task. In the Stop Signal Task (SST; Logan, 1994) adolescents were instructed to respond to go trials but inhibit their response on stop trials. The outcome was the stop signal reaction time (SSRT), the mean time required to inhibit responses to stop trials as calculated by the integration method (Logan, 1994). A shorter SSRT reflects stronger inhibition.

2.2.4.2 Self-report and teacher report. The Inhibit subscale of the Behavioral Rating Inventory of Executive Functions Self Report and Teacher Report (BRIEF-SR and BRIEFTR; Gioia, Isquith, Guy, \& Kenworthy, 2000) consisted of nine items. The outcome measure was the total score, with higher scores reflecting more problems in inhibition.

\subsubsection{ToM.}

2.2.5.1 Experimental task. The Hinting Task (Corcoran et al., 1995) consisted of ten short read aloud stories. All stories ended with a hint about what a character wants the other to do and adolescents were asked what the character really meant when he/she said this. The outcome measure was the total score, with higher total scores reflecting better ToM.

2.2.5.2 Self-report. The Basic Empathy Scale (BES; Jolliffe \& Farrington, 2006) consisted of 20 items construing a cognitive and an affective empathy subscale. We analysed these subscales separately as cognitive empathy (i.e., understanding of other's emotions) may be more directly related to ToM than affective empathy (i.e., empathizing with others' emotions). The outcome measures were mean subscale scores, with higher scores reflecting better ToM.

2.2.5.3 Teacher report. In the Mindful Conversational Difficulties Scale (MCDS; De Rosnay et al., 2014) teachers answered eight questions about adolescents' ToM behavior 
compared to TD adolescents from a similar age. The outcome was the mean score, with higher scores indicating more difficulties with ToM. Therefore, scores were multiplied by -1 to obtain an index of capacity instead of impairment.

\subsubsection{Negative interpretation bias.}

2.2.6.1 Experimental task. The Interpretation Recognition Task (IREC-T; Houtkamp, van der Molen, de Voogd, Salemink, \& Klein, 2017) consisted of seven selected social scenarios. Adolescents were instructed to indicate the likelihood of a negative interpretation at the end of each ambiguous scenario. The outcome was the mean score, with higher scores reflecting stronger negative interpretations.

2.2.6.2 Self-report. The Social Anxiety Scale for Children - Revised (SASC-R; La Greca \& Stone, 1993) consisted of 22 items construing three subscales: Fear of Negative Evaluation (FNE) and two Social Avoidance and Distress (SAD) subscales. We analysed the FNE subscale separately and combined the SAD subscales as the FNE subscale may be more directly related to negative interpretation bias than the SAD subscales. The outcomes were the sum scores, with higher scores indicating more symptoms.

\subsection{Procedure}

Data collection ran from October 2018 until March 13 $3^{\text {th }}, 2020$ (it ended at the start of the country's lockdown due to COVID-19 pandemic). After providing consent, one of the parents digitally filled out the parent reports. Adolescents were tested individually in a quiet room at school and were assisted by a research assistant. As the test battery was large, test administration was divided over two sessions of 90 minutes, each with a short break halfway. The BART peer and solo conditions were counterbalanced over the two sessions to control for potential learning effects. This led to an A and B version of the test protocol (see Supplement 
A part XII). There was a minimum of two days and a maximum of four weeks between the sessions. At the end of the second session, adolescents were paid $€ 8$,- plus the money earned in one of the two randomly selected BART conditions (total approximately €16,-). Teachers were approached via email to fill out teacher reports. After completion of the data collection, both adolescents and parents were debriefed about the peer manipulation by phone. To get an indication of the credibility of the BART peer manipulation, we asked adolescents to rate the credibility from 0 to 100 .

\subsection{Data analysis}

Most analyses were preregistered, non-preregistered analyses are explicitly mentioned as exploratory. Bonferroni corrected $p$-values $\left(p^{B}\right)$ were calculated by multiplying $p$-values of comparisons belonging to the same construct by the number of comparisons.

2.4.1 Group comparisons. To assess differences between adolescents with and without MBID, we performed group comparisons. We deviated slightly from our first preregistration as we performed ANCOVA's instead of ANOVA's. Due to the COVID-19 related lockdown, the TD group was significantly older and included relatively more boys than the MBID group (see Table 1). Therefore, age and sex were added as covariates. The assumption of homogeneous regression lines was met as age and sex did not interact with group (MBID vs. TD) on all outcomes. This indicates that potential group differences were not influenced by age and were not different for boys and girls. We calculated partial eta squared effect sizes (denoted by $\eta_{p}{ }^{2}$ ). Although we did not reach the sample size of our preregistered power analysis, a post-hoc power calculation using $\mathrm{G}^{*}$ Power (Faul et al., 2007) based on ANCOVA's with a medium effect size $(f=.25)$ and $\alpha=.025, .016$ or $.0125(\alpha=.05$ divided by two, three or four indicators for Bonferroni correction) showed that our power was still $.82, .77$, or .74 respectively. 
2.4.2 SEM. To investigate how inhibition, ToM and negative interpretation bias were linked to susceptibility to peer influence in adolescents with MBID, we fitted a SEM only in this group. The indicators were the standardized experimental, self-, and teacher report scores ${ }^{3}$ and the latent factors were susceptibility to peer influence, inhibition, ToM and negative interpretation bias. We estimated these latent factors based on multiple indicators measuring the same construct and linked the latent factors amongst each other. For identification of the model, we fixed the variances of latent factors and the factor loading of the BART difference score to the susceptibility to peer influence factor to equal one.

The number of free parameters in the model is 18 , meaning we would need 90 adolescents to adhere to the rule of 5 participants per parameter (Kaplan, 2008). An a-priori power analysis based on medium effect sizes, 4 latent variables, and 12 indicators, showed that 137 participants were sufficient to achieve a power of .80 (Soper, 2021). Our preregistered SEM resulted in model non-identification, potentially due to the current sample size being smaller than intended $\left(N_{M B I D}=111\right)$. We solved this problem by fixing the residual variances of the indicators to a value of .25 , which translates to $75 \%$ of the total variance in each standardized indicator being explained by its latent factor. This reflects our expectation that the indicators were good indicators of the latent variable. Analyses were run in Mplus (Version 7.31) using maximum likelihood estimation.

\subsubsection{Secondary and exploratory analyses (see Supplement B parts II and III for}

results). As preregistered secondary analyses, we tested whether psychopathology as indicated on the SDQ moderated the group comparisons and we performed within-construct correlations between experimental tasks, self- or teacher-reports in the MBID and TD group

\footnotetext{
${ }^{3}$ Standardizing the scores has the advantage of knowing the variance of the indicators, namely it being equal to 1. This allows us to easily fix the residual variance in terms of 'proportion of total observed variance', which is necessary due to the small sample size relative to the number of free model parameters. We report unstandardized results as a default. Standardized results are only reported if at least one of the hypothesized relations between the latent factors is observed.
} 
separately. As exploratory analyses, we tested group differences on the SDQ subscales, average BART peer manipulation credibility and we reran the group comparisons with only a selection of the MBID group (i.e., adolescents with Borderline Intellectual Functioning $(70<\mathrm{IQ}<85)$ and adolescents with (possible) deficient adaptive functioning). 


\section{Results}

\subsection{Preliminary analyses}

From the 196 recruited adolescents, 33 adolescents were excluded (e.g., IQ did not match inclusion criteria), leaving a sample of 163 adolescents. Concerning the test scores, $3.5 \%$ were missing, $1.1 \%$ had errors (e.g., SSRT lower than 100 ms which indicates unreliable estimation), and $0.8 \%$ were outlying based on Median Absolute Deviation exceeding 2.5 (Leys et al., 2013). Adolescents with missing, erroneous or outlying data were excluded from the corresponding group comparisons. See details in Supplement B part I. For the SEM, full information maximum likelihood (FIML) was used to handle missing data ${ }^{4}$.

Adolescents with MBID had lower scores on IQ, BSA-j, and SES relative to TD adolescents, while they had more 'possible' or 'likely' psychopathology as measured with the SDQ total difficulties score (see Table 1). In the total sample, the number of pumps was higher in the BART peer condition $(M=48.46, S D=11.47)$ compared to the BART solo condition $(M=43.67, S D=11.17, t(162)=-6.88, p<.001, d=.54$; see Figure $1 \mathrm{~A})$, suggesting an effective peer manipulation.

\subsection{Group comparisons}

Regarding susceptibility to peer influence, adolescents with and without MBID did not differ on the experimental task or the self-report (i.e., BART difference score see Figure 1A, and RPI). On inhibition, both groups did not differ on the experimental task, self-report and teacher-report (i.e., SST ${ }^{5}$, BRIEF-SR, and BRIEF-TR). Regarding ToM, adolescents with

\footnotetext{
${ }^{4}$ As some of our missing data are not missing at random (i.e., we removed outliers and preregistered errors), the FIML estimator may be considered inappropriate. Nevertheless, we refrained from excluding cases listwise as this leaves $N=74$ participants instead of $N=111$, translating to less than 5 individuals per free parameter.

${ }^{5}$ As the equal SSRT obtained by adolescents with MBID compared to TD adolescents may be due to adolescents with MBID responding slower on go trials, we compared the go reaction times (RT) and the number of go-trial errors with similar ANCOVA's as the other group comparisons. The mean go RT and the number of go-trial errors did not differ significantly between the two groups (both $p$ 's $>.29$ ).
} 
MBID had lower scores on the experimental Hinting task ${ }^{6}$ and on the BES Affective selfreport subscale than TD adolescents ${ }^{7}$ (see Figure $1 \mathrm{~B}$ and $1 \mathrm{C}$ ). This difference was not found on the BES Cognitive self-report subscale and on the MDCS teacher-report. For negative interpretation bias, groups did not differ on the experimental task and the self-report (i.e., IREC-T and SASC-R subscales ${ }^{6,7}$ ). In sum, we only observed differences between the MBID and TD group on two ToM instruments on which the MBID group showed decreased performance (see Table 3 for all group means and test results).

- Insert Figure 1 and Table 3 here -

\footnotetext{
${ }^{6}$ The homogeneity of variance assumption was violated for these variables. However, we made no adjustments to the analyses given that our group sample sizes were not deviating more than a ratio of 10 and as we already used a more stringent $\alpha$ level (Tabachnick \& Fidell, 2019).

${ }^{7}$ Sex was a significant covariate on the BES Affective subscale and both SASC-R subscales with girls reporting higher affective ToM and negative interpretation bias than boys.
} 


\subsection{SEM}

As adolescents with MBID were equally susceptible to peer influence as TD adolescents, and as knowledge about potential underlying mechanisms in MBID is limited, linking their susceptibility to peer influence on their inhibition, ToM and negative interpretation bias remains relevant. In the SEM on the MBID group, most indicators loaded positively on their proposed latent factor, except for the SST on inhibition ${ }^{8}$. This suggests that the latent factors represented the constructs they were intended to (squared multiple correlations per indicator are reported in Supplement B part IV). The latent factors of inhibition, ToM and negative interpretation bias were not significantly related to the susceptibility to peer influence factor (see Figure 2$)^{9,10}$. The fit indices suggest poor model fit (see Table 4). To check the robustness of these findings across analytical settings, we report two alternative SEMs demonstrating the same pattern of results in Supplement B part V.

- Insert Figure 2 and Table 4 here -

\footnotetext{
${ }^{8}$ In general, behavioral tasks such as the SST show low correlations with the BRIEF (see also Bramston \& Fogarty, 2000).

${ }^{9}$ In our preregistered model, the factor loading of the BART to susceptibility to peer influence was fixed to 1 to allow for identification. Therefore, the latent factor could only be informed by RPI scores, which complicated its interpretation. Exploratively, we fixed the variance of the latent factor to 1 instead. Both the BART and the RPI loaded significantly positive to peer influence factor. The pattern of relationships between the latent variables did not change.

${ }^{10}$ As covariances between latent constructs were low, we also fixed these to zero as a sensitivity check. Results were similar.
} 


\section{Discussion}

In this preregistered study, we used experimental tasks, and self- and teacher reports to investigate susceptibility to peer influence in adolescents with and without MBID. We expected higher susceptibility to peer influence in adolescents with MBID relative to TD adolescents. This hypothesis was not supported: groups did not differ in their susceptibility to peer influence on the experimental task and the self-report measure. Furthermore, and again contrary to our hypotheses, adolescents with MBID did not differ from TD adolescents on all measures of inhibition, negative interpretation bias, and on two of the four ToM measures. Group differences on the other two ToM measures were as expected: adolescents with MBID performed worse on the experimental ToM task and reported less affective empathy compared to TD adolescents. In the SEM within adolescents with MBID, all indicators, except the SST, loaded positively on the latent factors. The latent factors of inhibition, ToM and negative interpretation bias were not linked to the latent factor of susceptibility to peer influence.

\subsection{Comparing adolescents with and without MBID}

4.1.1 Susceptibility to peer influence. We found no evidence for heightened susceptibility to peer influence in adolescents with MBID compared to TD adolescents, which is not in line with earlier experimental and self-report research (Bexkens et al., 2019; Dekkers et al., 2017; Egger et al., 2021; Wagemaker et al., 2020). As peer influence situations are highly complex, susceptibility to peer influence of adolescents with MBID may depend on specific conditions such as the relation with the peers, the number of peers, and the exact peer influence used. To illustrate, in our experimental task, only one peer encouraged risk taking in two breaks of the task while earlier studies used multiple peers who encouraged risk taking regularly during the task (Bexkens et al., 2019; Wagemaker et al., 2020). An explanation for the different findings in the self-report could be that the difference in mean IQ between the 
MBID and TD samples was smaller in our study $\left(M_{\text {IQdiff }}=26.4\right)$ than in Dekkers et al. $\left(M_{\text {IQdiff }}\right.$ $=39.3$ ). Future experimental research could elucidate whether specific conditions or IQ criteria are crucial to find heightened susceptibility to peer influence in adolescents with MBID.

4.1.2 Inhibition. Adolescents with MBID were not different from TD adolescents with regard to inhibition. Our study was the first to use the experimental SST in adolescents with MBID. Although our null finding may seem counterintuitive, a meta-analysis in individuals with MBID also found that two of the five experimental studies on behavioral inhibition indices showed no significant differences (Bexkens, Ruzzano, et al., 2014). The BRIEF-SR has not been administered to adolescents with MBID specifically but a study using the BRIEF-TR in adolescents with and without MBID showed that more problems in behavioral regulation only depended on the presence of a behavioral disorder (Bexkens, Van Der Molen, et al., 2014). These findings suggest that inhibition differences found between adolescents with MBID and TD adolescents are variable.

4.1.3 ToM. Adolescents with MBID had weaker ToM compared to TD adolescents on some of our instruments. They had difficulties in interpreting subtle verbal hints, which is in line with earlier studies using ToM tasks (Baglio et al., 2016). They also reported more problems in empathizing with others' emotions than TD adolescents. Potentially, the abovementioned ToM aspects can be classified as complex ToM, which continues to develop during adolescence (Humphrey \& Dumontheil, 2016) and may be delayed in adolescents with MBID. A potential delay in ToM development in adolescents with MBID may also explain why adolescents with MBID were similar to TD adolescents on more basic forms of ToM such as understanding others' emotions.

4.1.4 Negative interpretation bias. The current study was the first to directly compare adolescents with and without MBID on their negative interpretation bias, showing no 
differences. This may indicate that the higher prevalence of social anxiety disorder in adolescents with MBID compared to TD adolescents (Dekker \& Koot, 2003) may not be related to the strength of a negative interpretation bias in adolescents with MBID. As adolescents with MBID have weaker cognitive control than TD adolescents (Bexkens, Ruzzano, et al., 2014 but cf. the current study), and as cognitive control plays a moderating role between negative interpretation bias and state anxiety (Salemink \& Wiers, 2012), a negative interpretation bias may instead have a bigger impact on anxious feelings in adolescents with MBID than in TD adolescents. Future research could elucidate links between negative interpretation bias, (social) anxiety and moderating factors in adolescents with MBID.

\subsection{Linking susceptibility to peer influence to inhibition, ToM, and negative interpretation bias}

This study indicated no link between susceptibility to peer influence of adolescents with MBID to their inhibition, ToM or negative interpretation bias. Future studies could differently operationalize the psychological processes that encode regulatory, socialcognitive, and affective cues from the environment (Do et al., 2020). If these null findings remain replicated, research on susceptibility to peer influence in adolescents with MBID should target different concepts. A relevant target for investigation may be the influence of deviant friends. That is, adolescents with MBID more often have a deviant peer group than TD adolescents (Tipton et al., 2013), which may therefore bring them more often into problematic peer influence situations. Interventions aimed at decreasing susceptibility to peer influence should not target inhibition, ToM or negative interpretation bias, but may better target susceptibility to peer influence directly. As an example, a curriculum for adolescents 
with developmental disorders may be promising, as it improved decision-making in hypothetical peer influence situations (Khemka et al., 2016).

\subsection{Strengths}

The current study has several strengths. First, the entire study was preregistered, giving confidence in fair treatment of the data (Nosek et al., 2015). We acknowledge that we did not reach our preregistered sample size due to the COVID-19 pandemic. Nevertheless, we still managed to recruit a large sample of adolescents with MBID, a group that is complex and hard to recruit. Second, our multimethod approach enabled us to make more reliable claims about the constructs based on multiple instruments. We combined multiple indicators of one construct in a latent factor in the SEM, which removed noise and increased reliability compared to using single indicators. Third, although instrument selection for research in adolescents with MBID can be challenging, we selected our instruments based on explicit criteria, adapted all self-reports with comprehension checks, and piloted two instruments which we adapted accordingly. Fourth, debriefing credibility questions showed that the peer manipulation was credible.

\subsection{Limitations}

A first limitation is that we may have studied a relatively mild MBID sample. Adolescents with MBID were sampled from practical vocational track schools that only admit students with an IQ between 55 and 80, which we confirmed by a shortened IQ test, and learning delays. However, teachers rated adaptive functioning of $41 \%$ of our MBID sample as sufficient. Thus, potentially $41 \%$ of our MBID sample did not meet the criteria of MBID. An explanation for this could be that we only used a screener of adaptive functioning. During our study, the Dutch version of the Adaptive Behavior Assessment System became available (i.e., 
ABAS-3; Harrison \& Oakland, 2020). This more extensive instrument may be used in future research to get more detailed information on adaptive functioning.

Second, a limitation of our experimental tasks of inhibition, ToM and negative interpretation bias could be that they provided too little emotional load. Situations of peer influence are usually highly emotional for adolescents (Gunther Moor et al., 2014), suggesting that factors involved should also be measured with such an emotional load. First versions of inhibition tasks using emotional faces have been developed (e.g., Pawliczek et al., 2013). For ToM or negative interpretation bias, virtual reality (e.g., Otkhmezuri et al., 2019) can be used to increase emotional load.

Third, it might be posited that our SEM was underpowered to observe latent links between susceptibility to peer influence and inhibition, ToM, and negative interpretation bias. However, we found that the magnitude of these links was very small, suggesting that even in larger samples they will not become significant.

\subsection{Conclusions}

Our study showed that adolescents with MBID do not differ from TD adolescents in their susceptibility to peer influence as well as in many other domains and that their inhibition, ToM, and negative interpretation bias were not related to their susceptibility to peer influence. The increased understanding of susceptibility to peer influence in adolescents with MBID offers fruitful areas for future peer influence research and interventions to support this specific group. 


\section{References}

American Psychiatric Association. (2013). Diagnostic and Statistical Manual of Mental Disorders (DSM-5®). American Psyciatric Association.

Baglio, G., Blasi, V., Intra, F. S., Castelli, I., Massaro, D., Baglio, F., Valle, A., Zanette, M., \& Marchetti, A. (2016). Social competence in children with borderline intellectual functioning: Delayed development of theory of mind across all complexity levels. Frontiers in Psychology, 7, 1-10. https://doi.org/10.3389/fpsyg.2016.01604

Bexkens, A., Huizenga, H. M., Neville, D. A., Collot d'Escury-Koenigs, A. L., Bredman, J. C., Wagemaker, E., \& Van der Molen, M. W. (2019). Peer-Influence on Risk-Taking in Male Adolescents with Mild to Borderline Intellectual Disabilities and/or Behavior Disorders. Journal of Abnormal Child Psychology, 47(3), 543-555. https://doi.org/10.1007/s10802-018-0448-0

Bexkens, A., Ruzzano, L., Collot d' Escury-Koenigs, A. M. L., Van der Molen, M. W., \& Huizenga, H. M. (2014). Inhibition deficits in individuals with intellectual disability: A meta-regression analysis. Journal of Intellectual Disability Research, 58(1), 3-16. https://doi.org/10.1111/jir.12068

Bexkens, A., Van Der Molen, M. W., Collot Descury-Koenigs, A. M. L., \& Huizenga, H. M. (2014). Interference control in adolescents with Mild-to-Borderline Intellectual Disabilities and/or behavior disorders. Child Neuropsychology, 20(4), 398-414. https://doi.org/10.1080/09297049.2013.799643

Blakemore, S. J. (2018). Avoiding Social Risk in Adolescence. Current Directions in Psychological Science, 27(2), 116-122. https://doi.org/10.1177/0963721417738144

Bramston, P., \& Fogarty, G. (2000). The assessment of emotional distress experienced by people with an intellectual disability: A study of different methodologies. Research in Developmental Disabilities, 21(6), 487-500. https://doi.org/10.1016/S08914222(00)00054-8 
Brechwald, W. A., \& Prinstein, M. J. (2011). Beyond homophily: A decade of advances in understanding peer influence processes. Journal of Research on Adolescence, 21(1), 166-179. https://doi.org/10.1111/j.1532-7795.2010.00721.x

Brewer, J., \& Hunter, A. (2006). Foundations of multimethod research: synthesizing styles. Sage.

Corcoran, R., Mercer, G., \& Frith, C. D. (1995). Schizophrenia, symptomatology and social inference: Investigating "theory of mind" in people with schizophrenia. Schizophrenia Research, 17(1), 5-13. https://doi.org/10.1016/0920-9964(95)00024-G

Dekker, M. C., \& Koot, H. M. (2003). DSM-IV disorders in children with borderline to moderate intellectual disability. I: Prevalence and impact. Journal of the American Academy of Child and Adolescent Psychiatry, 42(8), 915-922. https://doi.org/10.1097/01.CHI.0000046892.27264.1A

Dekkers, L. M. S., Bexkens, A., Hofman, A. D., Boeck, P. De, Collot d'Escury, A. L., \& Huizenga, H. M. (2017). Formal Modeling of the Resistance to Peer Influence Questionnaire. Assessment. https://doi.org/10.1177/1073191117698754

Do, K. T., Prinstein, M. J., \& Telzer, E. H. (2020). Neurobiological susceptibility to peer influence in adolescence. In Handbook of Developmental Cognitive Neuroscience. https://www.researchgate.net/publication/332655929

Egger, S., Nicolay, P., Huber, C., \& Müller, C. M. (2021). Increased openness to external influences in adolescents with intellectual disability: Insights from an experimental study on social judgments. Research in Developmental Disabilities, 113, 103918. https://doi.org/10.1016/j.ridd.2021.103918

Emerson, E., Felce, D., \& Stancliffe, R. J. (2013). Issues Concerning Self-Report Data and Population-Based Data Sets Involving People With Intellectual Disabilities. Intellectual and Developmental Disabilities, 51(5), 333-348. https://doi.org/10.1352/1934-9556- 


\section{5 .333}

Faul, F., Erdfelder, E., Lang, A.-G. G., \& Buchner, A. (2007). G*Power 3: A flexible statistical power analysis program for the social, behavioral, and biomedical sciences. Behavior Research Methods, 39(2), 175-191. https://doi.org/10.3758/BF03193146

Gioia, G. A., Isquith, P. K., Guy, S. C., \& Kenworthy, L. (2000). Behavior Rating Inventory of Executive Function. Psychological Assessment Resources.

Goodman, R., Ford, T., Simmons, H., Gatward, R., \& Meltzer, H. (2000). Using the Strengths and Difficulties Questionnaire (SDQ) to screen for child psychiatric disorders in a community sample. British Journal of Psychiatry, 177(6), 534-539. https://doi.org/10.1192/bjp.177.6.534

Gunther Moor, B., Bos, M. G. N., Crone, E. A., \& Van der Molen, M. W. (2014). Peer rejection cues induce cardiac slowing after transition into adolescence. Developmental Psychology, 50(3), 947-955. https://doi.org/10.1037/a0033842

Guy, S. C., Isquith, P. K., \& Gioia, G. A. (2004). Behavior rating inventory of executive function-Self-report version: Professional Manual. Psychological Assessment Resources, Inc.

Harrison, P. L., \& Oakland, T. (2020). ABAS-3 Schaal voor adaptief gedrag/Scale for adaptive behavior. Hogrefe.

Houtkamp, E. O., van der Molen, M. J., de Voogd, E. L., Salemink, E., \& Klein, A. M. (2017). The relation between social anxiety and biased interpretations in adolescents with mild intellectual disabilities. Research in Developmental Disabilities, 67, 94-98. https://doi.org/10.1016/j.ridd.2017.06.003

Humphrey, G., \& Dumontheil, I. (2016). Development of Risk-Taking, Perspective-Taking, and Inhibitory Control During Adolescence. Developmental Neuropsychology, 41(1-2), 59-76. https://doi.org/10.1080/87565641.2016.1161764 
Kaplan, D. (2008). Structural equation modeling: foundations and extensions (Vol. 10). Sage Publications.

Khemka, I., Hickson, L., \& Mallory, S. B. (2016). Evaluation of a Decision-Making Curriculum for Teaching Adolescents with Disabilities to Resist Negative Peer Pressure. Journal of Autism and Developmental Disorders, 46(7), 2372-2384. https://doi.org/10.1007/s10803-016-2770-0

Klein, A. M., Houtkamp, E. O., Salemink, E., Baartmans, J. M. D., Rinck, M., \& van der Molen, M. J. (2018). Differences between self- and peer-rated likability in relation to social anxiety and depression in adolescents with mild intellectual disabilities. Research in Developmental Disabilities, 80, 44-51. https://doi.org/10.1016/j.ridd.2018.05.016

La Greca, A. M., \& Stone, W. L. (1993). Social Anxiety Scale for Children-Revised: Factor Structure and Concurrent Validity. Journal of Clinical Child Psychology, 22(1), 17-27. https://doi.org/10.1207/s15374424jccp2201_2

Laghi, F., Bianchi, D., Pompili, S., Lonigro, A., \& Baiocco, R. (2019). Cognitive and affective empathy in binge drinking adolescents: Does empathy moderate the effect of self-efficacy in resisting peer pressure to drink? Addictive Behaviors, 89, 229-235. https://doi.org/10.1016/j.addbeh.2018.10.015

Lejuez, C. W., Richards, J. B., Read, J. P., Kahler, C. W., Ramsey, S. E., Stuart, G. L., Strong, D. R., \& Brown, R. A. (2002). Evaluation of a behavioral measure of risk taking: The balloon analogue risk task (BART). Journal of Experimental Psychology: Applied, 8(2), 75-84. https://doi.org/10.1037/1076-898X.8.2.75

Lekkerkerker, L., \& Konijn, C. (2011). Beoordeling sociaal aanpassingsvermogen bij jeugdigen/Assessment of Social Adaptive Functioning in Adolescents. Kind En Adolescent, 32(3), 134-145.

Leys, C., Ley, C., Klein, O., Bernard, P., \& Licata, L. (2013). Detecting outliers: Do not use 
standard deviation around the mean, use absolute deviation around the median. Journal of Experimental Social Psychology, 49(4), 764-766.

https://doi.org/10.1016/J.JESP.2013.03.013

Meldrum, R. C., Miller, H. V., \& Flexon, J. L. (2013). Susceptibility to Peer Influence, SelfControl, and Delinquency. Sociological Inquiry, 83(1), 106-129. https://doi.org/10.1111/j.1475-682x.2012.00434.x

Miers, A. C., Blöte, A. W., Bögels, S. M., \& Westenberg, P. M. (2008). Interpretation bias and social anxiety in adolescents. Journal of Anxiety Disorders, 22(8), 1462-1471. https://doi.org/10.1016/j.janxdis.2008.02.010

MPLUS Version 7.3.1 [Computer Software]. (n.d.). Muthén \& Mtuhén.

Na, S. D., \& Burns, T. G. (2016). Wechsler Intelligence Scale for Children-V: Test Review. In Applied Neuropsychology: Child (Vol. 5, Issue 2, pp. 156-160). Routledge. https://doi.org/10.1080/21622965.2015.1015337

Nigg, J. T. (2000). On inhibition/disinhibition in developmental psychopathology: Views from cognitive and personality psychology and a working inhibition taxonomy. Psychological Bulletin, 126(2), 220-246. https://doi.org/10.1037/0033-2909.126.2.220

Nosek, B. A., Aarts, A. A., Anderson, C. J., Anderson, J. E., Barry Kappes, H., \& Barry, H. (2015). Open Science Collaboration - Estimating the reproducibility of psychological science. Science, 349(6251), aac4716-aac4716. https://doi.org/10.1126/science.aac4716

Otkhmezuri, B., Boffo, M., Siriaraya, P., Matsangidou, M., Wiers, R. W., Mackintosh, B., Ang, C. S., \& Salemink, E. (2019). Believing Is Seeing: A Proof-of-Concept Semiexperimental Study on Using Mobile Virtual Reality to Boost the Effects of Interpretation Bias Modification for Anxiety. JMIR Mental Health, 6(2), e11517. https://doi.org/10.2196/11517

Pawliczek, C. M., Derntl, B., Kellermann, T., Kohn, N., Gur, R. C., \& Habel, U. (2013). 
Inhibitory control and trait aggression: Neural and behavioral insights using the emotional stop signal task. NeuroImage, 79, 264-274.

https://doi.org/10.1016/j.neuroimage.2013.04.104

Salemink, E., \& Wiers, R. W. (2012). Adolescent threat-related interpretive bias and its modification: The moderating role of regulatory control. Behaviour Research and Therapy, 50(1), 40-46. https://doi.org/10.1016/j.brat.2011.10.006

Schreiber, J. B., Stage, F. K., King, J., Nora, A., \& Barlow, E. A. (2006). Reporting structural equation modeling and confirmatory factor analysis results: A review. In Journal of Educational Research (Vol. 99, Issue 6, pp. 323-338). Heldref. https://doi.org/10.3200/JOER.99.6.323-338

Simonoff, E., Pickles, A., Chadwick, O., Gringras, P., Wood, N., Higgins, S., Maney, J. A., Karia, N., Iqbal, H., \& Moore, A. (2006). The Croydon assessment of learning study: Prevalence and educational identification of mild mental retardation. Journal of Child Psychology and Psychiatry and Allied Disciplines, 47(8), 828-839. https://doi.org/10.1111/j.1469-7610.2006.01630.x

Soper, D. S. (2021). A-priori Sample Size Calculator for Structural Equation Models [Software]. https://www.danielsoper.com/statcalc

Stuijfzand, S., Creswell, C., Field, A. P., Pearcey, S., \& Dodd, H. (2018). Research Review: Is anxiety associated with negative interpretations of ambiguity in children and adolescents? A systematic review and meta-analysis. Journal of Child Psychology and Psychiatry and Allied Disciplines, 59(11), 1127-1142.

https://doi.org/10.1111/jcpp.12822

Tabachnick, B. G., \& Fidell, L. S. (2019). Using Multivariate Statistics (7th ed.). Pearson. Tipton, L. A., Christensen, L., \& Blacher, J. (2013). Friendship Quality in Adolescents with and without an Intellectual Disability. Journal of Applied Research in Intellectual 
Disabilities, 26(6), n/a-n/a. https://doi.org/10.1111/jar.12051

UNESCO. (1999). International Standard Classification of Education-ISCED. Author.

Wagemaker, E., Huizenga, H. M., Dekkers, T. J., Collot d'Escury-Koenigs, A., Salemink, E., \& Bexkens, A. (2020). When do those "risk-taking adolescents" take risks? The Combined Effects of Risk Encouragement by Peers, Mild-to-Borderline Intellectual Disability and Sex. Journal of Abnormal Child Psychology, 1-15. https://doi.org/10.1007/s10802-020-00617-8

Wechsler, D. (2008). Wechsler adult intelligence scale-Fourth Edition (WAIS-IV). Pearson. 
Table 1. Group Characteristics of Adolescents with MBID and TD Adolescents

\begin{tabular}{llll}
\hline & MBID $(\boldsymbol{n}=\mathbf{1 1 1})$ & TD $(\boldsymbol{n}=\mathbf{5 2})$ & Group comparisons \\
\hline Age & $15.91(.95)$ & $16.83(1.08)$ & $t(161)=5.51, p<.001$ \\
Sex (\% boys) & $55.9 \%$ & $76.9 \%$ & $\chi^{2}(1)=6.71, p=.01$ \\
IQ & $74.75(6.83)$ & $101.19(11.50)$ & $t\left(68.39^{\mathrm{a}}\right)=15.36, p<.001$ \\
Country of birth ${ }^{\mathrm{b}}$ (\% Dutch/other) & $87.5 \% / 12.5 \%$ & $100 \% / 0 \%$ & $\chi^{2}(1)=6.92, p=.01$ \\
BSA-jc (\% sufficient/potentially & $41.4 \% / 13.1 \% / 45.5 \%$ & $82.1 \% / 10.3 \% / 7.7 \%$ & $\chi^{2}(2)=20.39, p<.001$ \\
limited/probably limited) & & & \\
SDQ total (\% any disorder & $54.1 \% / 37.8 \% / 8.1 \%$ & $78.8 \% / 15.4 \% / 5.8 \%$ & $\chi^{2}(2)=9.60, p=.01$ \\
unlikely/possible/probable) & & & $t(133)=4.63, p<.001$ \\
SES $^{\mathrm{d}}$ & $4.76(.89)$ & $5.41(.54)$ & \\
\hline
\end{tabular}

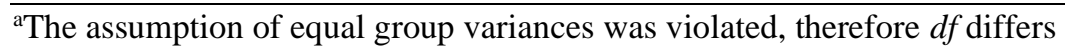

${ }^{\mathrm{b} C o u n t r y}$ of birth of 24 adolescents was not reported by parents (23 MBID, 1 TD)

${ }^{\mathrm{c} B S A-j}$ of 25 adolescents was not reported by teachers (12 MBID, 13 TD)

${ }^{\mathrm{d}} \mathrm{SES}$ of 28 parents was not reported (25 MBID, 3 TD)

Abbreviations: BSA-j Assessment of Social Adaptability - Youth, MBID Mild-to-Borderline Intellectual

Disability group, SDQ Strengths and Difficulties Questionnaire, TD Typically Developing control group

Table 2. Overview of Instruments

\begin{tabular}{|c|c|c|c|}
\hline Construct & Experimental task & Self-report & Teacher-report \\
\hline Susceptibility to & Balloon Analogue Risk & Resistance to Peer Influence & - \\
\hline peer influence & Task (BART) peer vs solo & Scale (RPI) & \\
\hline Inhibition & Stop Signal Task (SST) & $\begin{array}{l}\text { Behavioral Rating Inventory } \\
\text { of Executive Functions - Self } \\
\text { Report (BRIEF-SR) }\end{array}$ & $\begin{array}{l}\text { Behavioral Rating Inventory } \\
\text { of Executive Functions - } \\
\text { Teacher Report (BRIEF-TR) }\end{array}$ \\
\hline Theory of Mind & Hinting task & Basic Empathy Scale (BES) & $\begin{array}{l}\text { Mindful Conversational } \\
\text { Difficulties Scale (MCDS) }\end{array}$ \\
\hline $\begin{array}{l}\text { Negative } \\
\text { interpretation bias }\end{array}$ & Recognition task (IREC-T) & $\begin{array}{l}\text { Social Anxiety Scale for } \\
\text { Children - Revised (SASC-R) }\end{array}$ & - \\
\hline
\end{tabular}


Table 3. Group Comparisons between Adolescents with MBID and TD Adolescents: Means (M), Standard Deviations (SD's), and Test Results

\begin{tabular}{|c|c|c|c|c|c|c|}
\hline Construct & Instrument & $\operatorname{MBID} M(S D)$ & TD $M(S D)$ & MBID main effect & Sex effect & Age effect \\
\hline \multirow{2}{*}{$\begin{array}{l}\text { Susceptibility to } \\
\text { peer influence }\end{array}$} & BART DIF & $5.14(9.39)$ & $4.07(7.78)$ & $F(1,159)=.48, p^{B}=.98, \eta_{p}^{2}=.003$ & $F(1,159)=.23, p^{B}>1, \eta_{p^{2}}=.001$ & $F(1,159)=.14, p^{B}>1, \eta_{p^{2}}=.001$ \\
\hline & RPI & $-29.81(5.69)$ & $-30.13(4.89)$ & $F(1,159)=.83, p^{B}=.73, \eta_{p}^{2}=.01$ & $F(1,159)=.69, p^{B}=.82, \eta_{p^{2}}=.004$ & $F(1,159)=1.28, p^{B}=.52, \eta_{p}^{2}=.01$ \\
\hline \multirow{3}{*}{ Inhibition } & SST & $221.09(47.17)$ & $221.98(43.90)$ & $F(1,140)=.08, p^{B}>1, \eta_{p^{2}}=.001$ & $F(1,140)=1.31, p^{B}=.76, \eta_{p}^{2}=.01$ & $F(1,140)=2.22, p^{B}=.42, \eta_{p^{2}}=.02$ \\
\hline & BRIEF-SR & $14.44(3.45)$ & $15.76(3.13)$ & $F(1,158)=4.08, p^{B}=.14, \eta_{p^{2}}=.03$ & $F(1,158)=1.54, p^{B}=.65, \eta_{p^{2}}=.01$ & $F(1,158)=.16, p^{B}>1, \eta_{p^{2}}=.001$ \\
\hline & BRIEF-TR & $16.08(5.57)$ & $13.92(4.38)$ & $F(1,133)=3.67, p^{B}=.17, \eta_{p^{2}}=.03$ & $F(1,133)=1.88, p^{B}=.52, \eta_{p^{2}}=.01$ & $F(1,133)=.55, p^{B}>1, \eta_{p^{2}}=.004$ \\
\hline \multirow{4}{*}{ ToM } & Hinting task & $17.18(1.73)$ & $18.94(1.12)$ & $F(1,154)=37.25, p^{B}<.001^{* *}, \eta_{p^{2}}=.20$ & $F(1,154)=2.24, p^{B}=.55, \eta_{p}^{2}=.01$ & $F(1,154)=.13, p^{B}>1, \eta_{p^{2}}=.001$ \\
\hline & $\mathrm{BES}-\mathrm{C}$ & $3.86(.58)$ & $4.07(.51)$ & $F(1,159)=3.69, p^{B}=.23, \eta_{p^{2}}=.02$ & $F(1,159)=3.39, p^{B}=.27, \eta_{p^{2}}=.02$ & $F(1,159)=1.27, p^{B}>1, \eta_{p^{2}}=.01$ \\
\hline & $\mathrm{BES}-\mathrm{A}$ & $2.92(.85)$ & $3.24(.80)$ & $F(1,159)=8.16, p^{B}=.02^{*}, \eta_{p^{2}}=.05$ & $F(1,159)=25.10, p^{B}<.001^{* *}, \eta_{p^{2}}=.14$ & $F(1,159)=.54, p^{B}>1, \eta_{p^{2}}=.003$ \\
\hline & MDCS & $-2.99(.46)$ & $-2.68(.43)$ & $F(1,134)=5.22, p^{B}=.10, \eta_{p^{2}}=.04$ & $F(1,134)=.22, p^{B}>1, \eta_{p^{2}}=.002$ & $F(1,134)=2.78, p^{B}=.39, \eta_{p^{2}}=.02$ \\
\hline \multirow{3}{*}{$\begin{array}{l}\text { Negative } \\
\text { interpretation } \\
\text { bias }\end{array}$} & IREC-T & $17.42(3.98)$ & $18.04(3.42)$ & $F(1,147)=3.07, p^{B}=.25, \eta_{p^{2}}=.02$ & $F(1,147)=5.73, p^{B}=.05, \eta_{p}^{2}=.04$ & $F(1,147)=1.50, p^{B}=.67, \eta_{p^{2}}=.01$ \\
\hline & SASC-R FNE & $1.73(.71)$ & $1.86(.58)$ & $F(1,154)=4.99, p^{B}=.08, \eta_{p^{2}}=.03$ & $F(1,154)=19.26, p^{B}<.001^{* *}, \eta_{p}^{2}=.11$ & $F(1,154)=.83, p^{B}>1, \eta_{p^{2}}=.01$ \\
\hline & SASC-R SAD & $2.07(.77)$ & $2.04(.58)$ & $F(1,156)=.34, p^{B}>1, \eta_{p^{2}}=.002$ & $F(1,156)=11.24, p^{B}=.003^{* *}, \eta_{p}^{2}=.07$ & $F(1,156)=.20, p^{B}>1, \eta_{p^{2}}=.001$ \\
\hline
\end{tabular}

Note: $* p^{B}<.05, * * p^{B}<.01$

Abbreviations: BART DIF Balloon Analogue Risk Task difference score, BES-C/-A Basic Empathy Scale - Cognitive and Affective subscale, BRIEF-SR/-TR Inhibit subscale of the Behavioral Rating Inventory of Executive Functions Self Report and Teacher Report, IREC-T Interpretation Recognition Task, MBID Mild-to-Borderline Intellectual Disability group, MDCS Mindful Conversational Difficulties Scale, RPI Resistance to Peer Influence Scale, SASC-R FNE / SAD Social Anxiety Scale for Children - Revised Fear of Negative Evaluation and Social Avoidance and Distress subscales, SST Stop Signal Task, TD Typically Developing control group, ToM Theory of Mind 
Table 4. Fit Indices of the SEM

\begin{tabular}{lcc}
\hline Fit index & Cut-off for good fit & Value \\
\hline $\mathrm{X}^{2}$ & $p>.05$ & $p<.0001$ \\
$\mathrm{CFI}$ & $\geq .90$ & .000 \\
$\mathrm{TLI}$ & $\geq 0.95$ & -3.52 \\
RMSEA & $<0.08$ & .34 \\
SRMR & $<0.08$ & .23 \\
\hline
\end{tabular}

Abbreviations: $\mathrm{X}^{2}$ Chi-square, TLI Tucker Lewis Index, CFI Comparative Fit Index, RMSEA Root Mean Square

Error of Approximation, SRMR Standardized Root Mean Square Residual 